\title{
Robot Mediated Handholding Combined with a Mobile Video Call Makes the Users Feel Nearer and Closer
}

\author{
Kazuaki Tanaka \\ Kyoto Institute of Technology \\ k_tanaka@kit.ac.jp
}

\author{
Reo Mayuzumi \\ Kyoto Institute of Technology
}

\author{
Tomomi Takahashi \\ Kyoto Institute of Technology
}

\author{
Sho Takaki \\ Kyoto Institute of Technology
}

\author{
Natsuki Oka \\ Kyoto Institute of Technology
}

\section{KEYWORDS}

Social touch, Robotic hand, Handholding, Telepresence, Social bonding

\section{ACM Reference Format:}

Kazuaki Tanaka, Reo Mayuzumi, Tomomi Takahashi, Sho Takaki, and Natsuki Oka. 2021. Robot Mediated Handholding Combined with a Mobile Video Call Makes the Users Feel Nearer and Closer. In Proceedings of the 9th International Conference on Human-Agent Interaction (HAI '21), November 09-11, 2021, Virtual Event, Japan. ACM, New York, NY, USA, 10 pages. https://doi.org/10.1145/3472307.3484168

tion, positional inconsistency also happens. This study developed robotic hand-type social touch device and investigated whether it enhances the sense of space sharing and social bonding even though these inconsistencies occur. Experimental results revealed that the user's belief that the finger movements of the device were the partner's hand movement contributed to the sense of space sharing. The movements of the device according to the flow of conversation facilitated this belief; hence, the sense of space sharing increased in spite of the inconsistencies. Positional inconsistency was also found to have an adverse effect on social bonding. Positional consistency contributes to enhance social bonding because it visually facilitates the user's belief that the device is a substitute for the partner's body. Basically, the functions of the device, such as skin softness, body temperature, finger movements, and grip force, which enhance the reality of the touch sensation, could be effective in facilitating the user's beliefs. However, it was suggested that awareness of temperature does not always make positive contribution to the reality and could lead to eeriness.

\section{CCS CONCEPTS}

- Human-centered computing $\rightarrow$ Collaborative and social computing; Collaborative and social computing devices; • Hardware $\rightarrow$ Communication hardware, interfaces and storage; Tactile and hand-based interfaces; Haptic devices.

Permission to make digital or hard copies of all or part of this work for personal or classroom use is granted without fee provided that copies are not made or distributed for profit or commercial advantage and that copies bear this notice and the full citation on the first page. Copyrights for components of this work owned by others than ACM must be honored. Abstracting with credit is permitted. To copy otherwise, or republish, to post on servers or to redistribute to lists, requires prior specific permission and/or a fee. Request permissions from permissions@acm.org.

HAI '21, November 09-11, 2021, Virtual Event, Japan

(C) 2021 Association for Computing Machinery.

ACM ISBN 978-1-4503-8620-3/21/11 ..\$15.00

https://doi.org/10.1145/3472307.3484168

\section{INTRODUCTION}

Generally, social bonds for maintaining and building close relationships with family members, lovers, friends, etc. are weakened when living in a place away from them. This issue has led to depression due to loneliness when assigned to work alone, divorce due to loneliness of not being able to meet, and a decrease in the success rate of long-distance relationships. Recently, the self-restraint of movement by COVID-19 has exacerbated this problem.

To solve it, in the field of telepresence, techniques have been developed that bring conversations with remote partners closer to face-to-face conversations. The basic approach is to present the appearance and behavior of partners in a high-definition life-size video calling [1]. Currently, because $4 \mathrm{~K}$ and $8 \mathrm{~K}$ large LCD TVs are widespread, making video calls with extremely high-definition images is possible. However, there exists a limit to the approach to high-definition video because it is difficult to recognize the difference even if the definition is further improved. In addition, regardless of the image quality, it is possible that the conversation itself through the display makes the partners feel distant. The mirror neurons that are activated when a monkey sees human behavior differ depending on whether they are close to or far from a person. It is known that when a monkey sees human behavior through a transparent board, neurons are activated in the same way as when it is far from the person [2]. Even in the case of humans, if the same brain function occurs, it is possible that the perception that the display hinders the feeling of being with a conversation partner in the same space.

Recently, an approach has been proposed that combines telerobotics with video calls. As one of them, the effect of reproducing shaking hands with a remote partner with a robotic hand has been verified; it is known that the remote hand shaking enhances the feeling of being in the same space as the partner (space sharing) [3]. This result indicates that reproducing social touch with another person may have changed the perception of talking with a 


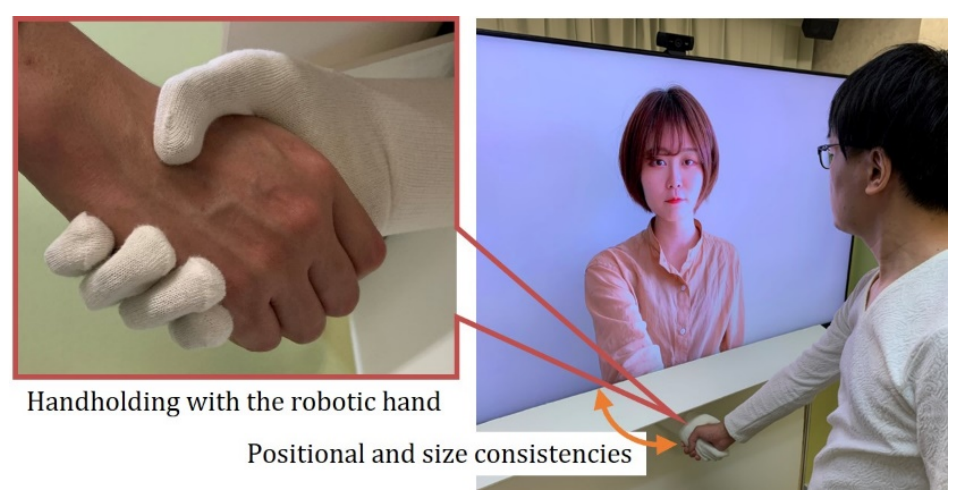

Figure 1: Positional and size consistencies between the life-size partner's video and the robotic hand.

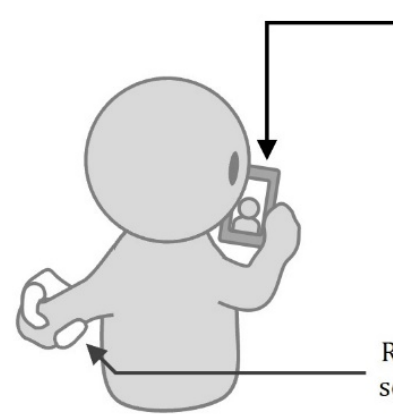

a) Separated Design:

Positional and size inconsistencies occur
Video call on a mobile phone

Robotic hand-type social touch device

b) Combined Design:

Only size inconsistency occurs

Figure 2: Positional and size inconsistencies between the reduced partner's video and the social touch device.

remote partner through the display. The study also shows that even a handshake reproduced by a robotic hand can provide intimacy; therefore, it is expected that reproducing social touch will enhance social bonds. According to the findings of this previous study, as shown in Figure 1, the robotic hand should be installed on the extension of the partner's arm, so as to make the partner's life-size image and the robotic hand in the real space to be positionally consistent. This consistency is effective in enhancing the sense of space sharing. Furthermore, since the previous study used a life-size image and a full-scale robotic hand, the size consistency may also be effective. However, with the spread of smartphones, many users are now making video calls on small displays. In the reduced video call, it is difficult to provide positional and size consistencies to the combination of the partner's video and the social touch device.

As shown in Figure 2, when combining a mobile phone such as a smartphone with a robotic hand-type social touch device, the following two designs can be considered: a) separated design, in which the mobile phone is operated with the right hand while the touch device is held with the left hand, and b) combined design, in which a mobile phone is attached to the device and held with the right hand. In the separated design, the touch device can be connected to the mobile phone via a general wireless communication standard such as Bluetooth, so that the function of mediating social touch can be added regardless of the shape of the mobile phone. However, although the user faces the partner's image, the touch device simulates the state of holding the hand of the partner who is positioned to the immediate left. Therefore, positional inconsistency occurred in the separated design. In contrast, the combined design reduces positional inconsistency because the touch device simulates the state holding the hand of the partner who faces the user. However, it is necessary to design a touch device to fit the shape of the mobile phone. Furthermore, size inconsistency occurs in both designs owing to the reduced video. Positional or size inconsistencies may decrease the reality of social touch with a partner. Although such inconsistencies occur, if the conditions under which the reproducing social touch enhances the sense of space sharing and social bonding are clarified, it can be expected to become the design guidelines for social touch devices that expand mobile phones. To clarify this condition, this study developed a robotic hand-type social touch device, and investigated how the positional and size inconsistencies that occur when the device is combined with a video call on a mobile phone influences the sense of space sharing and social bonding.

\section{RELATED WORKS}

Studies on remote touch have long history. Previous studies have proposed various devices that add the function of remote touch to existing remote communication media, such as text chats, voice and 
video calls, etc. A commonly used haptic stimulus in such devices is vibration [4-6]. For example, when a user squeezes a mobile device, the partner's device vibrates [4]. Other stimuli include the force of rotation with doorknob-type [7] and roller-type [8] devices, tightening with bands attached to a wrist [9] and an arm [10], poking a cheek with a pneumatically inflated silicone balloon [11], and joystick force feedback [12], etc. Body touch is known to be an effective modality for emotional communication [13]. Most of these studies used haptic stimulus as a modality to convey nonverbal information because text chats and voice calls cannot transmit the users' facial expressions and emotional gestures. Therefore, these previous studies evaluated whether a remote partner could correctly decode the non-verbal information encoded as a haptic stimulus, but the reality of the reproduced touch sensation was not focused on.

In contrast, previous studies on remote touch in video calls have addressed the reality of reproduced touch sensations with the aim of supporting intimate interaction. First, in a previous study that reproduced a handshake [3], the necessity of showing the user that the robotic hand is moving in synchronization with the user's hand has been verified. When showing a partner's hand on a display to demonstrate synchronization between the human and robotic hands, the hand showing on the display and the robotic hand in the real space are redundant. The recognition of the body duplication reduces reality. To enhance the sense of space sharing, it therefore is effective to create positional consistency with the robotic hand by showing the partner's image without including the part beyond their wrist in the view angle rather than visually showing the synchronization. Regarding enhancing intimacy, no differences have been reported with or without positional consistency. As a touch device that reproduces handshake, a band-type device that tightens the user's hand and presents their body temperature has also been proposed [14]. This previous study investigated the effect of remote touch on collaborative work in the media space, but the effect of enhancing the sense of space sharing was not significant. When users place their hands on the corresponding place in the shared space, this device tightens each other's hands to reproduce a handshake. In this method, there exists positional inconsistency in that the user's hand is held by the partner even though the partner's hand is placed on the desk. As a result, the reality of body contact decreased, and hence, the sense of space sharing may have decreased.

In addition to handshakes, devices that reproduce kisses and hugs have also been proposed. The device that mediates kissing reproduces the pressure and movement of the partner's lips with servomotors [15]. This previous study mentioned that the device creates a more intimate interaction compared to ordinary video calls. However, even with this device, the effect of enhancing the sense of space sharing has not been obtained. As separated from the display for a video call, this device suffers from positional inconsistency. The kissing device that solves the positional inconsistency by allowing a mobile phone to be attached to the device was also been proposed later [16]. However, it has not been verified whether the solution enhances the sense of space sharing. In addition, size inconsistency still occurs in this improved device.

Devices that reproduce hugs have been proposed in many previous studies, some of which are clothing-type [17-19]. Clothing-type devices with multiple oscillators have been proposed for use in combination with mirror-type video calls [18]. This method simulates a conversation while looking at a mirror that shows the hugging users. The combination of a clothes-type device with multiple oscillators and a video call has also been reported in previous studies. When the user touches the touch display showing the partner's image, the corresponding part of the jacket worn by the partner vibrates [20]. These studies have evaluated whether the feeling of hugging with the other party can be obtained, or whether the position touched by the other party can be correctly recognized, but they have not investigated the effects of increasing these evaluations on the sense of space sharing and social bonding.

In a previous study that verified the effect of reproducing a hug, a voice call made while hugging a bolster with a built-in mobile phone was proposed [21]. The study reported that this method reduces stress hormones in saliva compared to normal voice calls. A hug that cannot see the partner's face is compatible with voice calls, and so there is no need to consider the positional and size inconsistencies. However, it is not clear whether the effect of hugging a bolster is superior to that of video calling, which allows one to talk while looking at the other person. This study will clarify whether the combination of a social touch device with a video call has a positive effect on video calls regardless of positional and size inconsistencies.

\section{DEVELOPMENT OF THE ROBOTIC HAND-TYPE SOCIAL TOUCH DEVICE}

This section describes the mechanism of our robotic hand-type social touch device developed to create a realistic haptic sensation. This device reproduces finger flexion and extension movements, body temperature, and skin softness. A schematic diagram of the remote touch system developed in this study is illustrated in Figure 3 , while the structure of the device is demonstrated in Figure 4. As shown in Figure 3, this system is asymmetrical between remote places to facilitate the experiment described in Section 4. If the same device is prepared in both places between remote areas, it is possible to hold the hands together bidirectionally.

\subsection{Finger Movements}

The human finger joints are the DIP joint at the tip (called the IP joint in the case of the thumb), the PIP joint in the middle, and the MP joint at the root. To reproduce the finger movement while holding a partner's hand, it is not necessary to control all joints independently, but the degree of flexion of each finger must be controlled separately. Therefore, each of the five servomotors controls one finger. The mechanism for finger flexion and extension is shown in the upper part of Figure 4. The strings that act as tendons run above and below the finger, and are connected to the circular horn of the servomotor. For these strings, we used a fishing line called the PE line, which has high strength and low elongation. Rotate the horn, pull the below string (red), loosen the above string (blue) to flex the finger, and rotate it in the opposite direction to extend it. The physical limits of joint flexion were designed to be $90^{\circ}$ for the DIP, PIP, and IP joints, and $45^{\circ}$ for MP joints so that the subjects' hands were not pressed with excessive grip force. Owing to these flexion limits, we confirmed that even if the five fingers were completely flexed, there was sufficient space between the fingers 


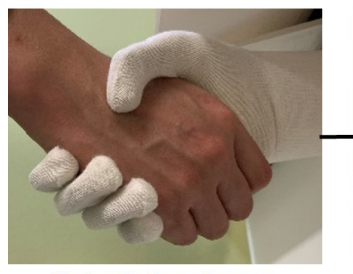

Robotic hand-type social touch device

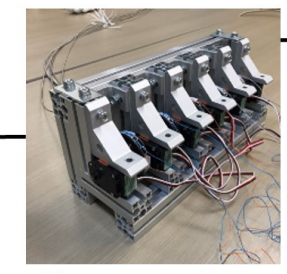

Five servomotors

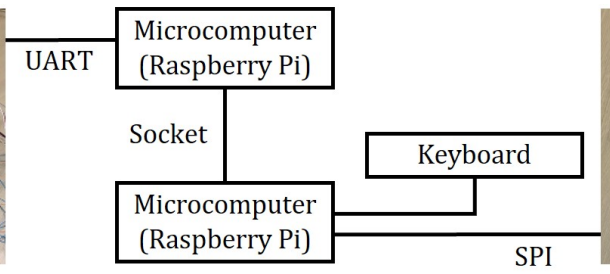

SPI

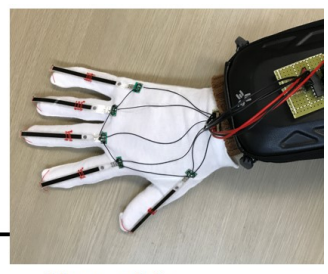

Glove with

the bending sensors

Figure 3: Schematic diagram of the remote social touch system using our robotic hand-type social touch device.

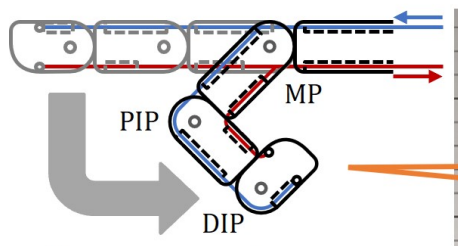

Finger movements: Controlled by pulling the strings with servomotors

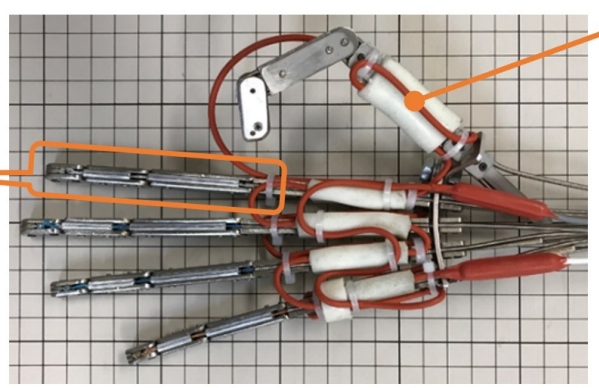

Body temperature:

Wiring the silicon cord heater

Skin softness:

Covering with urethane gel material

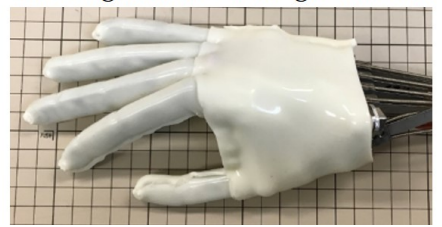

Figure 4: Structure of our robotic hand-type social touch device.

and the palm, and it was possible for a person to easily pull out their hand. The finger bones are made of stainless steel (SUS304), and the material of the wrist parts that fix the finger bones is aluminum (A6063). The size of the skeleton of the device was designed to be close to the average hand size of a Japanese female because a female experimenter operated the device in the experiment.

\subsection{Body Temperature}

To reproduce the body temperature, a one-meter silicon cord heater was placed along the palm part of the bones (the middle part of Figure 4). To keep the temperature of the device constant, a temperature controller executes ON/OFF control based on sensing with a temperature sensor ( $\mathrm{K}$ thermocouple), which is also placed on the palm part. Temperature perception is caused by the temperature difference between the skin and touch object. If the temperature of the device and the subjects' palm are approximately the same, it is expected that they will not notice the temperature of the device. Since the temperature of the human palm is around $33^{\circ} \mathrm{C}[22]$, the temperature of the device was set to $37{ }^{\circ} \mathrm{C}$, which is sufficiently higher than that.

\subsection{Skin Softness}

As shown in the lower part of Figure 4, to reproduce the skin softness, the skeleton of the device was covered with a urethane resin sheet (Asker C hardness 0 ). The softness of this material is close to that of human skin, but its surface has a sticky rubber-like texture, which is very different from the texture of human skin. Because it was difficult to reproduce the texture of the skin, we decided to wear thin cloth gloves on the device and reproduce the touch sensation of holding the gloved hand.

\section{EXPERIMENT}

To investigate the effects of positional and size inconsistencies caused by combining a social touch device with a video call, a subject experiment was conducted using a robotic hand-type social touch device. During the experiment, a female experimenter and a male subject interacted with each other, assuming a situation in which a man and a woman make a video call on a mobile phone. Twelve students (20-23 years old) from our university participated as test subjects.

\subsection{Conditions}

The three experimental conditions, namely the video, separate, and combined conditions, are illustrated in Figure 5. The details of each condition are as follows.

Video condition (baseline): The experimenter and the subject were seated in separate rooms and made a video call via Skype using the front mobile phone placed vertically on the stand.

Separated condition (positional and size inconsistencies occur): In addition to the situation of video conditions, the subject held the robotic hand-type social touch device with their left hand. Therefore, the device simulated the situation in which the subject was holding the right hand of the partner sitting to the left side. Against this situation, since the video of the partner was displayed on the mobile phone in front, there was positional inconsistency. In addition, while the video was a reduced image, the device imitated a life-sized hand, which caused size inconsistency.

Combined condition (only the size inconsistency occurs): In addition to the situation of video conditions, the subject held the robotic hand-type social touch device with their right hand. Therefore, the device simulated the situation in which the subject 


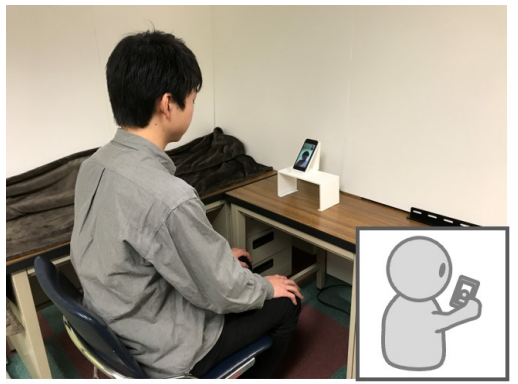

Video condition

(Baseline)

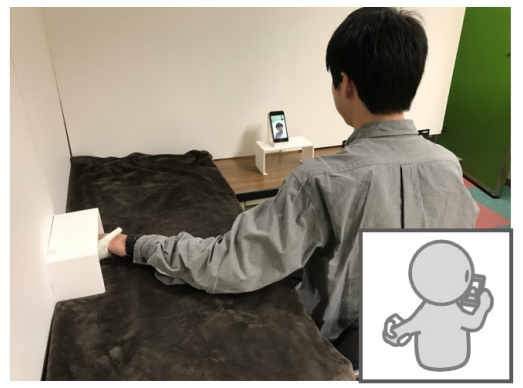

Separated condition

(The positional and size inconsistencies)

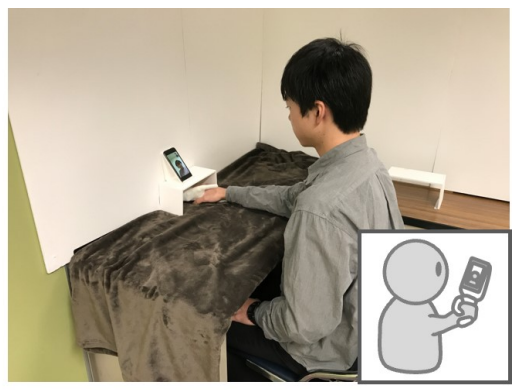

Combined condition

(Only the size inconsistencies occur)

Figure 5: Experimental conditions and environments.

was holding the right hand of the partner sitting in front. Since the video of the partner was displayed on the mobile phone in front, there was no positional inconsistency, but size inconsistency still existed.

\subsection{Procedure}

The experiment was a within-subject design; therefore, one subject experienced all the conditions. The order in which the three conditions were performed was different for all 12 subjects to counterbalance. First, a different experimenter (hereinafter referred to as an instructor) from the experimenter who played the remote conversation partner guided the subject to the seat. In the video condition, the instructor pointed to the mobile phone and explained to the subject, "This time, you will use this smartphone to talk with a person in another room." In the separated and combined conditions, the instructor pointed to the robotic hand-type social touch device and the mobile phone, with an explanation to the subject saying "This time, you will use this smartphone to talk with a person in another room while holding this hand".

The video call was connected before the subject was seated, while the camera on the experimenter's side was turned off so that they could not see the video of the experimenter. After that, when the instructor said, "Then, please start to talk", the experimenter turned on the camera and started the conversation. Once started, the instructor left a position that was invisible to the subject.

The topics of the three conversations were movie theaters, theme parks, and zoos, with their order being fixed. The experimenter led the conversation, and her lines were decided in advance. Table 1 shows an example of a conversation between the experimenter and the subject. The degree to which the preference matches with the other person may affect their intimacy with them. To control the degree of matching preferences between the conditions regardless of the topics, the experimenter agreed with the subjects as to whether or not they would often go to the place, and said her favorite one (movie genres, attractions, and animals), which was different from the one the subject answered. We prepared two favorite things and the reasons so that the experimenter could choose a favorite one different from the subjects'.

In the separated and combined conditions, the experimenter operated the device with the keyboard during the conversation. There were three types of operations: "holding", "holding firmly", and "opening". First, the "holding" operation was performed at the same time as the conversation started. Then, the "holding firmly" operation was performed on the shaded area in Table 1. The number of the "holding firmly" operation was set to seven under the separated and combined conditions. Finally, at the end of the conversation, an "opening" operation was performed. The "holding" and "opening" movements were the pre-recorded experimenter's hand movements by using the bending sensor shown in Figure 3 (the synchronization between a human hand and the device: https://youtu.be/PXeVxIJ9TR0). The "holding firmly" movement is to add a random value to the command values of all servomotors from the "holding" state to make holding the subjects' hand slightly stronger.

At the end of the conversation, the instructor said, "Thank you for cooperating the Xth (X was 1 to 3 ) conversation. Now, we will prepare for the next conversation, so please sit down and wait," and lead the subject to a waiting space away from the experimental space. After completing all three conditions, the instructor asked the subjects to answer the questionnaire described in the next section, and then conducted an interview.

\subsection{Questionnaire}

The questionnaire was conducted to investigate the effects of remote social touch on mobile video calls. The participants responded to each item on a 7-point Likert scale $(1=$ strongly disagree, $4=$ neither agree nor disagree, $7=$ strongly agree). In addition, the subjects described the reasons for the responses in the free description field.

First, the following items were set to confirm that the subjects did not feel any difference between the conditions in the quality of the video call.

- I felt the partner's utterance was easy to hear.

- I felt the video was clear enough.

A previous study of remote touch [3] used the expression "the feeling of talking in the same room" to evaluate the sense of space sharing. This expression was also employed in this work as well. We also asked about the feeling that the partner is next to or in front of the subject because it is possible that the subject's expectation of the position where the partner exists may differ depending on the 
Table 1: Conversation example between the experimenter and the subject (the experimenter performs the "holding firmly" operation on the shaded area)

\begin{tabular}{|c|c|c|}
\hline Speaker & Utterance (in Japanese) & Translation into English \\
\hline \multirow[t]{3}{*}{ Experimenter } & こんにちは。よろしくお願いします。 & Hello. Nice to meet you. \\
\hline & $\begin{array}{l}\text { 今回もあなたのことを色々と教えていただき } \\
\text { たいと思っています. }\end{array}$ & $\begin{array}{l}\text { I would like you to tell me a lot about you this } \\
\text { time as well. }\end{array}$ \\
\hline & あなたは動物園にはよく行きますか？ & Do you often go to the zoo? \\
\hline Subject & はい,たまに行きます。 & Yes, I go there once in a while. \\
\hline \multirow[t]{3}{*}{ Experimenter } & そうなんですね。 & Great. \\
\hline & 私も動物が好きで，よく行くんですよ。 & I also like animals and I often go there. \\
\hline & あなたの好きな動物ってなんでしょうか？ & What is your favorite animal? \\
\hline Subject & サルが好きです。 & I like monkeys. \\
\hline \multirow[t]{2}{*}{ Experimenter } & そうなんですね. & Really. \\
\hline & サルのどんなところが好きなんですか？ & What do you like about monkeys? \\
\hline Subject & サルは動きが面白いところが好きです。 & I like the funny movements of monkeys. \\
\hline \multirow[t]{5}{*}{ Experimenter } & $\begin{array}{l}\text { あ一確かに結構機敏な動きをするイメージあ } \\
\text { りますね. }\end{array}$ & $\begin{array}{l}\text { Ah, I have the impression that monkeys are } \\
\text { likely to move swiftly. }\end{array}$ \\
\hline & 私はパンダが好きです。 & I like pandas. \\
\hline & $\begin{array}{l}\text { まんまるな体でのんびり笹を食べている姿が } \\
\text { とっても可愛いんですよ. }\end{array}$ & $\begin{array}{l}\text { Their round body and appearance when eating } \\
\text { bamboo grass are very cute. }\end{array}$ \\
\hline & $\begin{array}{l}\text { 今回はあなたの好きな動物についておうかが } \\
\text { いできて良かったです. }\end{array}$ & $\begin{array}{l}\text { I'm glad to hear about your favorite animal this } \\
\text { time. }\end{array}$ \\
\hline & それでは，ありがとうございました. & Thank you. \\
\hline
\end{tabular}

physical embodiment and position of the robotic hand-type social touch device.

- I felt as if I were talking with the partner in the same room.

- I felt as if the partner were next to me.

- I felt as if the partner were in front of me.

It is known that giving intimacy through social touch enhances social bonding [23]. Therefore, previous studies on face-to-face social touch [24] and remote touch $[3,15]$ evaluated the degree of intimacy. In addition, since talking with a partner is necessary to enhance social bonding [25], asking the willingness to talk with the partner is also considered to be effective as an evaluation of social bonding [26]. For these reasons, the following three items were used for such evaluation:

- I felt intimacy with the partner.

- I enjoyed the conversation with the partner.

- I want to have more conversation with the partner.

Under the separated and combined conditions, it is thought that the more the device is regarded as the partner's hand, the stronger the sense of space sharing and social bonding. Since correlation analysis will be performed between these items and the evaluation values of space sharing and social bonding, the following items were set for the separated and combined conditions:

- I felt as if I were holding the partner's hand.

- I felt as if the partner were holding my hand.

The robotic hand-type social touch device produces skin softness, body temperature, finger movements, and grip force. The subjects ranked the four factors in the order they felt positive. To clarify which factors contribute to regard the device as the partner's hand, we also performed correlation analyses with the above two items in addition to comparing the average ranking of each factor. This investigation was conducted on a different paper after answering the questionnaire. Hereinafter, the subjects' feeling of "holding the partner's hand" and "being held their hand by the partner" are called the "holding sensation" and the "being held sensation", respectively.

\subsection{Results}

The obtained questionnaire results are illustrated in Figure 6. The bar graph shows the average value of each evaluation, while the error bar indicates the standard error. The three conditions were compared using one-way repeated-measures analysis of variance (ANOVA) followed by Bonferroni correction, whose results are shown in the figure. First, there existed no significant difference in the audio and video quality of the video calls between the conditions, which confirmed the control between the conditions regarding the quality of video calls.

Sense of space sharing: The feeling of talking in the same room $(F(2,22)=11.142, p<.001)$ was higher in the separated and combined conditions than in the video condition $(p<.05$, and $p$ $<.01$, respectively). The feeling that the partner was next to $(F(2$, 22) $=13.953, p<.001)$ was higher in the separated condition than in the video and combined conditions ( $p<.01$, both conditions). The feeling that the partner was in front $(F(2,22)=14.494, p<$ $.001)$ was higher in the video and combined conditions than in the separated condition $(p<.05$, and $p<.001$, respectively), and tended 


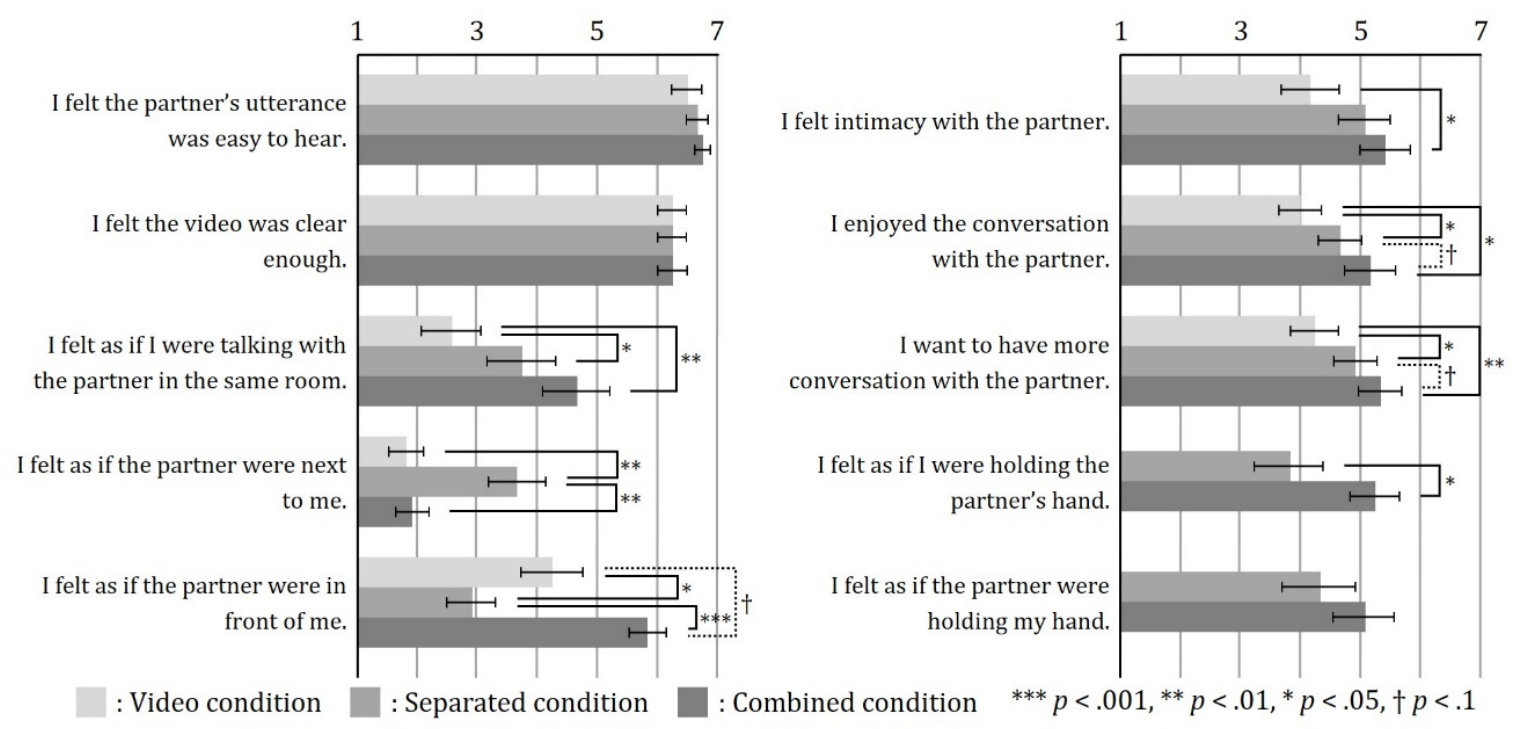

Figure 6: Questionnaire results.

Table 2: Correlation between the sense of space sharing, social bonding, as well as the holding and being held sensations

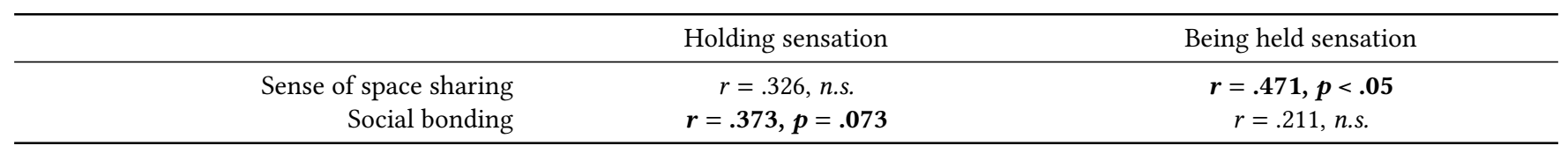

to be higher in the combined condition than in the video condition $(p=.081)$.

Social bonding: Intimacy with the partner $(F(2,22)=6.937$, $p<.01)$ was higher in the combined condition than in the video condition $(p<.05)$. The enjoyment of conversation $(F(2,22)=6.937$, $p<.01$ ) was higher in the separated and combined conditions than in the video condition ( $p<.05$, both conditions), and tended to be higher in the combined condition than in the separated condition $(p=.078)$. Similarly, the willingness to have more conversations $(F(2,22)=14.330, p<.001)$ was higher in the separated and combined conditions than in the video condition $(p<.05$, and $p<.01$, respectively), and tended to be higher in the combined condition than in the separated condition $(p=.052)$.

Beliefs regarding the device as the partner's hand: Since this evaluation was performed on separated and combined conditions, which were compared using the paired t-test. The holding sensation was higher in the combined than in the separated condition $(t(11)=2.837, p<.05)$, but there was no significant difference in the being held sensation. Correlation analysis was performed between these sensations and the sense of space sharing (the feeling of talking in the same room) and social bonding (intimacy with the partner). The results are shown in Table 2. The sense of space sharing had a significant positive correlation with the being held sensation $(r=.471, p<.05)$. Social bonding tended to have a weak positive correlation with the holding sensation $(r=.373, p=.073)$.

Effective factors of the robotic hand-type social touch device: The boxplot in Figure 7 shows the distribution of the order of the factors of the robotic hand-type social touch device that the subjects felt positive. As a result of comparing this ranking by the Friedman test, the difference between the factors was not significant $(\chi 2(3)=1.800, p=.615, n . s$.$) .$

In addition to this comparison, correlation analyses were performed between this ranking and the holding and being held sensations. To perform this analysis, ranks 1 to 4 were converted to values of 4 to 1 . The results are shown in Table 3. The being held sensation illustrated a significant positive correlation with finger movements $(r=.435, p<.05)$. The holding sensation was significantly negatively correlated with body temperature $(r=-.458, p<$ $.05)$, and tended to demonstrate a weak positive correlation with finger movements $(r=.347, p=.096)$.

\section{DISCUSSION}

\subsection{Sense of Space Sharing}

The experimental results revealed that holding hands mediated by the robotic hand-type social touch device enhanced the sense of space sharing in the video call on the mobile phone regardless of whether the partner's video and the device were separated or combined. This means that reproducing remote social touch enhances the sense of space sharing regardless of positional or size inconsistencies. In the separated condition that had positional inconsistency, although the partner's video was in front, subjects felt their partners were next to them because of the physical embodiment of the device. It is considered that the physical embodiment of 


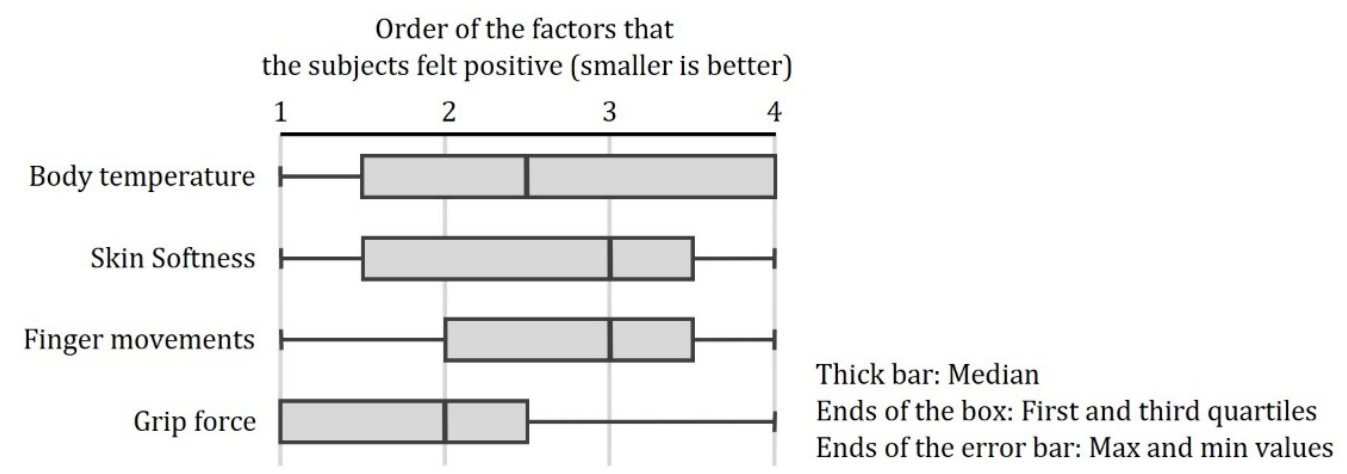

Figure 7: Distribution of the order of the factors of the robotic hand-type social touch device that subjects felt positive.

Table 3: Correlation between the holding and being held sensations and the ranking evaluation values of the factors of the robotic hand-type social touch device (the ranks 1 to 4 were converted to values of 4 to 1 )

\begin{tabular}{|c|c|c|}
\hline & Holding sensation & Being held sensation \\
\hline Body temperature & $r=-.458, p<.05$ & $r=-.269$, n.s. \\
\hline Skin Softness & $r=.092, n . s$. & $r=.073$, n.s. \\
\hline Finger movements & $r=.347, p=.096$ & $r=.435, p<.05$ \\
\hline Grip force & $r=.088, n . s$. & $r=-.196, n . s$. \\
\hline
\end{tabular}

the device had a stronger influence on the subjects' recognition of the partner's position than the video position, since the video was reduced on the small display. If the reality of touch sensations of the robotic hand-type social touch device can be further increased, the imagination that the partner is next to may be facilitated in voice calls without partner video.

The reason for the enhanced sense of space sharing regardless of positional and size inconsistencies can be described as follows: The sense of space sharing had a positive correlation with the being held sensation. Hence, it is possible that this sensation was responsible for enhancing the sense of space sharing. According to the subjects' free descriptions ( 3 of 12 subjects), this sensation was produced by the synchronization between the "holding firmly" movement of the device and the flow of conversation. The positive correlation found between this sensation and the ranking evaluation of the finger movements of the device corroborates this response. It is considered that this synchronization facilitated the subjects' belief that regards the device as the partner's hand, and as such, the remote social touch mediated by the device enhanced the sense of space sharing. Because the experimenter performed the "holding firmly" operation in both the separated and combined conditions, the difference in the being held sensation between these conditions was not significant. In the separated condition, it is thought that the being held sensation canceled the negative effect of positional inconsistency.

\subsection{Social Bonding}

Regarding social bonding (intimacy with the partner), only the combined condition was superior to the video condition. In addition, the enjoyment of the conversation and the willingness to talk to were enhanced in the separated and combined conditions; however, their evaluation values tended to be higher in the combined condition than in the separated one. From these results, it can be stated that reproducing remote social touch enhances social bonding regardless of size inconsistency, but which could be negatively affected by positional inconsistency.

Social bonding tended to be correlated with holding sensation, which was significantly higher in the combined condition than in the separated one; as such, the positive effect of positional consistency on this sensation was apparent. The positional consistency made it easier for the subjects to visually regard the device as the partner's hand; and thus, the remote social touch mediated by the device might enhance social bonding with the partner. The holding sensation was possibly contributed by not only the positional consistency but also the factors of the robotic hand-type social touch device. The next section focuses on this topic.

\subsection{Factors of the robotic hand-type Social Touch Device}

As discussed above, producing the holding and being held sensations could contribute to enhancing the sense of space sharing and social bonding, respectively. Therefore, we believe that increasing the reality of making the users regard the device as the partner's body part by reproducing movements and haptic sensations enhances the effects of the device that mediates social touch.

Since there was no difference in the ranking evaluation of the factors of the device, it is possible that all the factors, including skin softness, body temperature, finger movements, and grip force, worked effectively to produce the reality. Among them, finger movements were positively correlated with the being held sensation and 
tended to correlate with the holding sensation. Therefore, transmitting and reproducing the movement of a partner with a social touch device is considered to enhance the reality. However, it may not be effective unless the movement of the device is believed to be synchronizing with the movement of the partner. In a previous study on remote conversation via a robot, a control that makes subjects believe that the robot moves in synchronization with the partner's movement was performed prior to the conversation [27]. As discussed in Section 5.1, the fact that the finger movements of the device were according to the flow of conversation worked effectively to make this belief. The remote handshake conducted in a previous study was a short-time touch accompanied by a greeting [3]. In such cases, it was easy to make subjects believe in synchronization, even only by an experimenter's instruction. By contrast, when mediating longer-time social touch during conversation, as in this study, presenting the movements according to the conversation was necessary for making the belief.

Surprisingly, the ranking evaluation of body temperature was negatively correlated with the holding sensation. Three out of twelve subjects rated the temperature as number one, while another three rated it as number two, with many felt positive about body temperature. However, in the interview, despite the high ranking of body temperature, one subject said that he was surprised at the warmth while another mentioned about lukewarm and a little eerie. From these comments, it is possible that the awareness of body temperature caused the problem of the "uncanny valley", a phenomenon in which a more humanlike robot appearance gives an uncanny impression at a specific point [28]. The increase in haptic reality reproduced by the social touch device leads to a phenomenon similar to the reality of appearance.

As described in Section 3.2, temperature perception is caused by the temperature difference between the hand and object. In this study, the temperature of the device was set higher to be easily noticed by the subject. However, as varying from person to person, the temperature at which the user feels as if the device is a human hand could not be deterministic. Although awareness of temperature does not necessarily lead to the reality of touch sensation, a cold device would be felt mechanically. Hence, it may be effective to set the temperature to the same level or slightly higher than the user's hand.

\subsection{Limitations and Future Works}

In this study, the robotic hand-type device was designed based on the average hand size of a Japanese female, which was operated by one female experimenter. As such, it lacks verification corresponding to male operator. Future work should verify whether the negative effect of size inconsistency would occur unless the device size is designed according to the size of the male hand. In addition, in this study, the device mediated holding hands between the opposite sex; therefore, it is necessary to verify whether the same effect can be obtained between the same sex.

The temperature at which the user feels as if a social touch device is a part of the human body can depend on other factors of the device. The robotic hand-type social touch device used in this study wore a cloth glove. If the skin texture can be reproduced on the device surface, it may be easier to notice the body temperature even at lower temperature settings. In such cases, awareness of temperature is expected to increase the reality of touch sensations. Conversely, if the device does not have skin softness or reproduce body movements, it may not be felt as humanlike even when body temperatures are reproduced. Future work will aim to verify the interaction between the temperature and other factors of the social touch device and the relationship between the appropriate temperature difference between the user's hand and the device.

\section{CONCLUSION}

This study developed a robotic hand-type social touch device that mediates holding hands with a remote partner, and investigated the effect of positional and size inconsistencies caused by combining the device with a mobile video call. The results of the experiment illustrated that the device could enhance the sense of space sharing regardless of such inconsistencies, probably due to the feeling that the device movements correspond to the hand movements of the partner. Therefore, to facilitate this belief, operating the device according to the flow of conversation is considered to be effective for enhancing the sense of space sharing. The results also demonstrated that the device could enhance social bonding with the partner, regardless of size inconsistency, which, however, could be negatively affected by positional inconsistency. Therefore, making it easier for users to visually regard the device as a substitute for the partner's body through positional consistency might be effective in enhancing social bonding.

From these results, it can be stated that the interactions and interface designs that make the user feel as if the device were part of the partner body are effective in enhancing the sense of space sharing and social bonding. In addition, the reality of touch sensation of the device is also effective in supporting this belief. Our robotic hand-type social touch device increases this reality by reproducing the skin softness, body temperature, finger movements, and grip force. Among them, finger movements contribute to producing the reality that the partner is holding the user's hand. However, awareness of temperature does not always increase reality and could also cause eerie touch sensations. As such, it is necessary to investigate the relationship between temperature perception and the human likeness of the device.

It is expected that video calls with family members, lovers, and close friends will often be made on smartphones. The findings of this study serve as design guidelines for social touch devices used in combination with mobile phones. We hope that the findings will promote the development of social touch devices, and allow people living in remote locations to feel nearer and closer to each other.

\section{ACKNOWLEDGMENTS}

This work was supported by JSPS KAKENHI Grant Numbers JP19K12081, JP20H05564, JP21J15349.

\section{REFERENCES}

[1] Angela Prussog, Lothar Muhlbach, Martin Bocker. 1994. Telepresence in Videocommunications. Proc. Annual Meeting of Human Factors and Ergonomics Society. Vol.38, No.3, pp.180-184. https://doi.org/10.1177/154193129403800302

[2] Vittorio Caggiano, Leonardo Fogassi, Giacomo Rizzolatti, Peter Thier. 2009. Antonino Casile: Mirror Neurons Differentially Encode the Peripersonal and Extrapersonal Space of Monkeys. Science. Vol.324, No.5925, pp.403-406. https: //doi.org/10.1126/science.1166818 
[3] Hideyuki Nakanishi, Kazuaki Tanaka and Yuya Wada. 2014. Remote Handshaking: Touch Enhances Video-Mediated Social Telepresence. Proc. CHI2014. pp.21432152. https://doi.org/10.1145/2556288.2557169

[4] Angela Chang Sile O'Modhrain, Rob Jacob, Eric Gunther, Hiroshi Ishii. 2002. ComTouch: A Vibrotactile Communication Device. Proc. DIS2002. pp.312-320. https://doi.org/10.1145/778712.778755

[5] Kazi Masudul Alam, Abdulmotaleb El Saddik, Sandro Hardy, Aysha Akther. 2011 SMS Text Based Affective Haptic Application. Proc. VRIC2011.

[6] Masahiro Furukawa, Hiroyuki Kajimoto, Susumu Tachi. 2012. KUSUGURI: A Shared Tactile Interface for Bidirectional Tickling. Proc. AH2012. https://doi.org/ $10.1145 / 2160125.2160134$

[7] Jocelyn Smith, Karon MacLean. 2007. Communicating emotion through a haptic link: Design space and methodology. International Journal of Human-Computer Studies. Vol.65, No.4, pp.376-387. https://doi.org/10.1016/j.ijhcs.2006.11.006

[8] Scott Brave, Hiroshi Ishii, Andrew Dahley. 1998. Tangible Interfaces for Remote Collaboration and Communication. Proc.CSCW1998, pp.169-178. https://doi.org/ $10.1145 / 289444.289491$

[9] Katja Suhonen, Kaisa Vaananen-Vainio-Mattila, Kalle Makela. 2012. User Experiences and Expectations of Vibrotactile, Thermal and Squeeze Feedback in Interpersonal Communication. Proc. BCS-HCI2012. pp.205-214. https://doi.org/ 10.14236/ewic/HCI2012.26

[10] Rongrong Wang, Francis Quek. 2010. Touch \& Talk: Contextualizing Remote Touch for Affective Interaction. Proc. TEI2010. pp.13-20. https://doi.org/10.1145/ 1709886.1709891

[11] Young-Woo Park, Kyoung-Min Baek, Tek-Jin Nam. 2013. The Roles of Touch during Phone Conversations: Long-Distance Couples' Use of POKE in Their Homes. Proc. CHI2013. pp.1679-1688. https://doi.org/10.1145/2470654.2466222

[12] Jeremy N. Bailenson, Nick Yee, Scott Brave, Dan Merget, David Koslow. 2007 Virtual Interpersonal Touch: Expressing and Recognizing Emotions Through Haptic Devices. Human-Computer Interaction. Vol.22, No.3, pp.325-353. https: //doi.org/10.1080/07370020701493509

[13] Matthew J Hertenstein, Rachel Holmes, Margaret McCullough, Dacher Keltner. 2009. The Communication of Emotion via Touch. Emotion. Vol.9, No.4, pp.566-573. https://doi.org/10.1037/a0016108

[14] Svetlana Yarosh, Kenya Mejia, Baris Unver, Xizi Wang, Yuan Yao, Akin Campbell, Brad Holschuh. 2017. SqueezeBands: Mediated Social Touch Using Shape Memory Alloy Actuation. Proc. ACM on Human-Computer Interaction. https://doi.org/10 $1145 / 3134751$

[15] Hooman Aghaebrahimi Samani, Rahul Parsani, Lenis Tejada Rodriguez, Elham Saadatian, Kumudu Harshadeva Dissanayake, Adrian David Cheok. 2012. Kissenger: Design of a Kiss Transmission Device. Proc. DIS2012. pp.48-57. https://doi.org/10.1145/2317956.2317965

[16] Adrian David Cheok, Emma Yann Zhang. Electrical machine for remote kissing and engineering measurement of its remote communication effects, including modified Turing test. Journal of Future Robot Life. Vol.1, No.1, pp.111-134. 2020. https://doi.org/10.3233/FRL-200006

[17] Frank Vetere, eMartin R. Gibbs, Jesper Kjeldskov, Steve Howard, Florian F. Mueller, Sonja Pedell, Karen Mecoles, Marcus Bunyan. 2005. Mediating Intimacy: Designing Technologies to Support Strong-Tie Relationships. Proc. CHI2005. pp.471-480. https://doi.org/10.1145/1054972.1055038

[18] Osamu Morikawa, Sayuri Hashimoto, Tsunetsugu Munakata, Junzo Okunaka. 2006. Embrace System for Remote Counseling. Proc. ICMI2006. pp.318-325. https: //doi.org/10.1145/1180995.1181055

[19] James Keng Soon Teh, Adrian David Cheok, Roshan L. Peiris, Yongsoon Choi, Vuong Thuong, Sha Lai: Huggy Pajama. 2008. A Mobile Parent and Child Hugging Communication System. Proc. IDC2008. pp.250-257. https://doi.org/10.1145/ 1463689.1463763

[20] Longyu Zhang, Jamal Saboune, Abdulmotaleb El Saddik. 2015. Development of a haptic video chat system. Multimed Tools Appl 74. pp.5489-5512. https: //doi.org/10.1007/s11042-014-1865-x

[21] Hidenobu Sumioka, Aya Nakae, Ryota Kanai, Hiroshi Ishiguro. 2013. Huggable communication medium decreases cortisol levels. Scientific Reports. Vol.3, No.1. https://doi.org/10.1038/srep03034

[22] Sara E. Rimm-Kaufman, Jerome Kagan. 1996. The Psychological Significance of Changes in Skin Temperature. Motivation and Emotion. Vol.20, pp.63-78. https://doi.org/10.1007/BF02251007

[23] Sari M. van Anders, Katherine L. Goldey, Patty X. Kuo. 2011. The Steroid/Peptide Theory of Social Bonds: Integrating Testosterone and Peptide Responses for Classifying Social Behavioral Contexts. Psychoneuroendocrinology. Vol.36, No.9, pp.1265-1275. https://doi.org/10.1016/j.psyneuen.2011.06.001

[24] Valerian J. Derlega, Robin J. Lewis, Scott Harrison, Barbara A. Winstead, Robert Costanza. 1989. Gender differences in the initiation and attribution of tactile intimacy. Journal of Nonverbal Behavior. Vol.13, No.2, pp.83-96. https://doi.org/ 10.1007/BF00990792

[25] James C. McCroskey, J. Elaine Baer. 1985. Willingness to Communicate: The Construct and Its Measurement. Proc. the Annual Meeting of the Speech Communication Association.

[26] Naoki Koyama, Kazuaki Tanaka, Kohei Ogawa, Hiroshi Ishiguro. 2017. Emotional or Social?: How to Enhance Human-Robot Social Bonding. Proc. HAI2017. pp.203211. https://doi.org/10.1145/3125739.3125742

[27] Kazuaki Tanaka, Hideyuki Nakanishi, Hiroshi Ishiguro. 2015. Physical Embodiment Can Produce Robot Operator's Pseudo Presence. Frontiers in ICT. Vol.2, No.8. https://doi.org/10.3389/fict.2015.00008

[28] Masahiro Mori, Karl F. MacDorman, Norri Kageki. 2012. The Uncanny Valley. IEEE Robotics and Automation Magazine. Vol.19, No.2, pp.98-100. https://doi.org/ 10.1109/MRA.2012.2192811 